\title{
Research on the New and Innovative Environment and Landscape Planning Methodology based on the Concept of Green Building and Computer Aided Design Method
}

\author{
Tianfeng $\mathrm{Li}^{1}$ \\ ${ }^{1}$ College of Art and Design , \\ Nanyang institute of Technology, \\ Nanyang,Henan 473000,China
}

\begin{abstract}
In this paper, we conduct research on the new and innovative environment and landscape planning methodology based on the general concept of green building and computer aided design method. After the concept of green building shape to clear a set of advanced green building evaluation system has an important role in the sustainable development of our country's construction. Ecological restoration is a kind of compensation of nature in the construction of the city, if use to integrate landscape ecological restoration design, deterioration of ecological environment can be turned into a good ecological environment. Our solution performs well when solving the issues.
\end{abstract}

Keywords: Environment and Landscape; Green Building; Computer Aided Design.

\section{Introduction}

Construction is one of the largest consumers of resources industry, and its rapid development, make people realize that must go route of sustainable development in the field of construction. Green building is the very complicated system engineering, the development of green building has become a system, has vigorously promoted in developed countries, become the development direction of construction industry. The construction of green building evaluation system in our country lack of practical experience, at the primary stage, related evaluation system all draw lessons from foreign advanced experience. After the concept of green building shape, to clear a set of advanced green building evaluation system has an important role in the sustainable development of our country's construction, make our construction industry worth long-term study important issue. The emergence of green building marks the traditional architectural design from the only structure of architectural aesthetics, space utilization, form, color, structure, color and other considerations, gradually to look at from the perspective of ecological architecture, which means that the building is not only to as nonliving elements, and more is seen as the an integral part of the ecological circulation system. Green building is not only consider the local climate, the condition of architectural forms, using methods, facilities, construction process, construction materials and the good use of management, the influence of external environment and internal environment, comfortable, healthy at the same time considering the investors, customers, design, installation, operation and maintenance personnel of the interested. Can continue to design, good environment and benefit there should be a balance between and among users, benign interaction, so as to achieve the optimization of the overall greening effect.

The problems existing in the green building could be summarized as the follows. (1) Lack of the concept of green building in the construction project construction process, the concept of green building to carry out the inadequate. Displays in: the government did not emphasize the 
importance of green building, the designer will be green building indicators not fully used in the design, the builder is not fully consider the green building, the user didn't realize the value of building green and so on. In some developed regions, relatively to carry out the concepts of green building is better, and in less developed areas, from the structure of the decision to implement to use, cooperated-building parties and users are aware of the green building, still stay on the construction idea before. (2) Lack of advanced technology platform for the promotion now many buildings will be energy conservation and environmental protection technology to the building to live in, but there is no effective measure to promote and exchange platform, many advanced technology is not widely used. (3) A lack of laws and regulations from the concept of green building, since our country has been in the formulation of the relevant standards, but still lack of strong rules and regulations. From the design of the green building, green building materials selection, green building operations, lack of incentives to support system and the lack of restraint punishment, lead to land resources, water resources, energy-saving materials and other ecological resources. Through computer simulation technology, can be real 3D visualization of digital model in the city environment, the completion of effective urban planning and architectural design, no longer bound by the traditional method, the planning and architectural design staff as if in the scene, is advantageous to the best in the proper selection of design scheme, make the urban planning and architectural design for matching with the surrounding environment, but also can carry on the comparison of various schemes, to make a change to the design parameters, it can be in a real three-dimensional landscape environment will be the ideal design entity space effect [1].

To deal with the mentioned issues, in this paper, we conduct research on the new and innovative environment and landscape planning methodology based on the general concept of green building and computer aided design method. With the popularization of computer science application, computer has been thoroughly changed people's way of life. Even in some traditional industries, affected by computer technology deeply, and began to develop in the field of computer, to explore, to seek new survival. As garden project builders should have the courage to accept and learning new technology, keep up with the pace of The Times, positive reform engineering project management methods, to combine with the computer technology, so as to improve their scientific skills, at the same time, the project management on new starting point. In the next sections, we will discuss the issues in detail.

\section{Our Proposed New Methodology and Approach}

The Concept of Green Building. Green construction is a system topic engineering including the construction organization design, construction preparation, construction, general operation, and the equipment maintenance and construction site after the completion of the ecological restoration, etc. Required to reach a consensus of green construction, whole society to support and supervise the implementation of green construction, form a kind of social phenomenon. In planning and design stage, we should give full consideration to the overall requirements of green construction, to provide basic conditions for the green construction. Green construction management mainly includes the organization management, planning management, implementation, evaluation and personnel safety and health management. Organizational management main task is to build a green construction management system, and formulate the corresponding management system and the target. To prepare the green construction scheme planning management related regulations. The scheme should be read separately in the construction organization design, and in accordance with the relevant provisions of the 
examination and approval. Green construction plan should include environmental protection measures, material saving, water saving measures and energy saving measures, land and construction land protection measures, etc. When the blue prints and the design review materials saving and material resource utilization related content, to achieve material attrition rate is lower than norm attrition rate $30 \%$ according to the construction schedule, inventory situation and reasonable arrangement of material purchasing, play time and batch, reduce inventory as far as possible.

The site materials piled up and orderly, storage environment is appropriate, appropriate safekeeping measures. Material transport is appropriate, loading and unloading in the right way, prevent damage and spread, according to the site layout situation nearby uninstall, avoid and reduce the secondary material handling. Rain set in the construction site and the construction of sewage circulation channel, circulation channels through the grid connected to the sedimentation tank, settling basin for water treatment, can get a little suspended particles of water, water can be used again, to form the rain and the construction of sewage recycling. For more serious pollution and should not be repeated use, the construction of sewage and sewage biological ponds, dealing with people and then discharged into municipal pipe network. Green building for the people, architecture, natural environment and the social coordinated development as the goal, is represented by high efficiency, good environment and the sustainable development of architecture; Is embodied fully by using the basic principle of ecology, architecture and modern scientific means, take the initiative to adapt to the local ecological and does not destroy the ecological new ideas. Green buildings to give to life, it is a can actively interact with the environment, intelligent and adjustable system.

The Environment and Landscape Planning. With the rapid development of the economic construction in our country, the construction of modern city speed very big promotion. In the development of urban construction, the planning and design of the beautiful sex of the city, improve the working conditions of life in urban areas, is also an important project in urban development and construction, not only that, more can promote the development of urban planning and landscape design scientific and reasonable construction. Ecological restoration is a kind of compensation of nature in the construction of the city, if use to integrate landscape ecological restoration design, deterioration of ecological environment can be turned into a good ecological environment. But, in addition to natural factors, the urban environment is affected by the interference of man-made factors, so that the damaged ecosystem is difficult to implement resilience, or need very long time. In this case, we can use artificial design and engineering measures which can quickly restore the destroyed ecosystem and establish new urban green space landscape, the ecological restoration design for urban green space landscape planning has a certain practical significance [2-3].

Follow the principles of landscape ecological planning could be separated into the following parts. (1) The diversity and heterogeneity. In general, heterogeneity is an important attribute of urban green space landscape, if the greater the heterogeneity of urban green space landscape, urban green space landscape will present the diversity, its type will be more. In this case, the urban green space landscape has strong ability to prevent outside interference, to form stable ecological system, namely the heterogeneity and diversity maintenance in urban green space landscape planning plays a vital role. (2) Natural optimization and the overall optimization. For urban green space landscape ecological planning, must, analyzing the specific issues in strict accordance with the topography, rivers, and climate in different areas of the distribution, and plaques of city green space and ecological corridor, and open space reasonable layout, to 
reflect the ecological benefit, social benefit and economic benefit as the goal, optimize the natural state and the overall optimization of the urban green space landscape. (3) Continuity and integrity. In urban green space landscape planning, scientific application of landscape ecology, and emphasizes the restoration of green space landscape ecological pattern of the continuity and integrity. In terms of urban green space, is to maintain the natural green space in urban and suburban landscape patches between the contacts each other, and all this is done by the green corridor, namely urban green corridor planning must follow the principle of continuity and completeness of the landscape. (4) The general pattern of irreplaceability. In terms of ecological function, the pattern of urban green space landscape planning must have an alternative.

Landscape design of urban landscape planning, which is composed of complete ecosystem, has the certain integrity in terms of structure and function. In the process of urban landscape planning, need to cities, ecological environment and landscape design, landscape as a whole to consider and design. Urban landscape garden planning, it is necessary to take into account the overall premise, independent of unitized design. As a result, the city landscape garden planning has the characteristic of diversity. Because the natural environment has difference, natural landscape, formed under different environmental needs in the specific design conception through independent planning, to reflect the particular charm of unitized landscape. But because of the principle of diversity, often make the overall planning of urban landscape, often appear different design, unique unit of the landscape bright phenomena appear. It is the natural landscape culture and human culture common carrier system, is the foundation in urban landscape planning. The city landscape planning need to use the various thoughts of scientific knowledge, the proper use of city's natural resources, social resources, humanities resources, so the landscape design in urban landscape planning need to follow the basic principle of comprehensive. Landscape design need to reduce the cost of investment, through scientific and appropriate landscape design, clever use of city has the natural resources. It is the preferred way of landscape design and the last is the beautiful after adaptability, economy of garden design in beautiful to beautify the environment which has certain ornamental value. Urban ecological landscape planning in ecology, landscape architecture, such as geography subject developed on the basis of comprehensive and natural conservation, land planning and development, urban management of resources and environment are related. The significance of urban ecological landscape planning is to be able to let the in land use and development of the city has the rationality. In ecological landscape planning can be carried out within a certain area of science and the arrangement of resources development, effectively avoid the extensive use of resources, also protect the natural landscape and traditional city landscape is damaged which will let the citizens living environment improved. Effective out to protect the natural environment of urban ecological planning, landscape on the planning and design also has the aesthetic effect, make people when enjoy the material benefits can also be spiritual culture influence, and to enhance the quality of urban life.

The Computer Aided Design. Made static computer rendering process: first of all, on the computer for a certain object three-dimensional geometric model is set up, and then determine the three-dimensional geometric model of surface material, there are related in the scene illume and daylighting, selecting rational observation Angle, using computers to manipulate the rendering, made into a single static graphics, then displayed on a computer screen or through a printer to print on paper, the use of a single static graphics display, print software is simpler, can and rendering software in an independent state. Static 
rendering, for an object or a few buildings to express, the number of buildings more, only can only watch the panoramic view from the Angle of looking down. For some building number more and complex space form and the large range of urban landscape, or a more complex architecture interior landscape, more than just rely on static rendering image to the landscape, the object of attention fully displayed. By relying on nothing more than a few observations path with several animation, to complex urban space and architectural space landscape complete display, real-time dynamic landscape simulation and computer animation, by contrast, is more advanced. Degrees of freedom because the observer is greater than the animation, so the input of the object in the computer is more, can be simplified in dynamic and static rendering and increased the object three-dimensional geometric modeling workload, triggered a computer of geometric calculation and graphic data volume problem which will be meaningful.

\section{Conclusion}

In this paper, we conduct research on the new and innovative environment and landscape planning methodology based on the general concept of green building and computer aided design method. With the vigorous development of the computer network technology, more and more industries and fields have started to use the new computer network technology, at present the design of the architectural landscape associated with computer technology, in the process of drawing design, related design using computer aided. Therefore, under the background of innovation in the field of computer, design method of landscape architecture also is able to expand. Virtual reality technology is a comprehensive technology emerging in recent years, it is based on the development of computer technology, has been gradually applied to landscape architecture design, we firmly believe that in the recent future, the scope and the influence of its use in the future will be more extensive.

\section{References}

[1] Deng S L, Liu F Y, Yang M J, et al. Study on Computer Aided Design Method of Taper Leaf Spring[J]. Equipment Manufacturing Technology, 2014.

[2] Masson H, Arrighi P A. Method and system for designing a modeled assembly of at least one object in a computer-aided design system[D]. EP, 2011.

[3] Huang Z, Wang X, Hou Y. Novel Method of Fabricating Individual Trays for Maxillectomy Patients by Computer-Aided Design and Rapid Prototyping[J]. Journal of Prosthodontics,

2014. 